\title{
Myostatin induces mitochondrial metabolic alteration and typical apoptosis in cancer cells
}

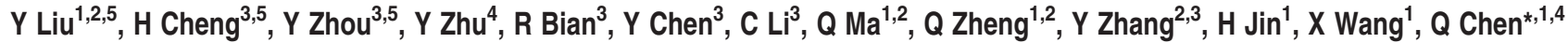 \\ and $\mathrm{D} \mathrm{Zhu^{*,2,3 }}$
}

Myostatin, a member of the transforming growth factor- $\beta$ superfamily, regulates the glucose metabolism of muscle cells, while dysregulated myostatin activity is associated with a number of metabolic disorders, including muscle cachexia, obesity and type II diabetes. We observed that myostatin induced significant mitochondrial metabolic alterations and prolonged exposure of myostatin induced mitochondria-dependent apoptosis in cancer cells addicted to glycolysis. To address the underlying mechanism, we found that the protein levels of Hexokinase II (HKII) and voltage-dependent anion channel 1 (VDAC1), two key regulators of glucose metabolisms as well as metabolic stress-induced apoptosis, were negatively correlated. In particular, VDAC1 was dramatically upregulated in cells that are sensitive to myostatin treatment whereas HKII was downregulated and dissociated from mitochondria. Myostatin promoted the translocation of Bax from cytosol to mitochondria, and knockdown of VDAC1 inhibited myostatin-induced Bax translocation and apoptosis. These apoptotic changes can be partially rescued by repletion of ATP, or by ectopic expression of HKII, suggesting that perturbation of mitochondrial metabolism is causally linked with subsequent apoptosis. Our findings reveal novel function of myostatin in regulating mitochondrial metabolism and apoptosis in cancer cells.

Cell Death and Disease (2013) 4, e494; doi:10.1038/cddis.2013.31; published online 14 February 2013

Subject Category: Cancer metabolism

Myostatin, a critical myokine and a member of the transforming growth factor- $\beta$ (TGF- $\beta$ ) superfamily, acts as a negative regulator of muscle mass ${ }^{1,2}$ and its mutation results in muscular hypertrophy and hyperplasia..,4 Like most Activin/ TGF- $\beta$ family members, myostatin binds to the cell surface activin receptor II or IIB (ACTRII, ACTRIIB), which recruits type I receptor ALK 4/5 (Activin receptor-like kinase 4 or 5 ) to form a complex. ${ }^{5,6}$ ALK5, which is also involved in the TGF- $\beta$ signaling pathway, could phosphorylate and activate Smad2/ 3 for its nuclear translocation and regulation of target genes transcription. ${ }^{7}$ Myostatin also utilizes the non-canonical pathway, such as activation of the MAPK pathway, or inhibition of the PI3K-Akt/GSK pathway, leading to suppression of myoblast proliferation and differentiation. ${ }^{8,9}$ Recent studies, including ours, have demonstrated that myostatin regulates glucose metabolism by promoting glucose consumption and uptake, increasing glycolysis and inhibiting glycogen synthesis in skeletal muscle cells. ${ }^{10,11}$

Myostatin circulates in the blood and its receptors are ubiquitously expressed in all tissues. Emerging evidence has suggested its function in regulating energy metabolism in both muscle and non-muscle cells. Knockout of myostatin in genetic mouse models of obesity and diabetes improved glucose metabolism and reduced obese phenotype. ${ }^{12}$ More specifically, it was found that myostatin treatment inhibited glucose uptake in placental cells. ${ }^{13}$ Despite these tantalizing results, it is possible that the reduction in adipose tissue mass in myostatin mutant mice is an indirect result of metabolic changes in skeletal muscle. ${ }^{14}$ It remains to be explored whether and how myostatin regulates metabolism in nonskeletal muscle tissues.

Accumulating evidence has also demonstrated that dysregulated myostatin is associated with metabolic disorders such as cachexia induced by tumors. ${ }^{15,16}$ As most cancer cells express myostatin receptors and several members of Activin/ TGF- $\beta$ family play very important roles in regulating cell growth, metabolism and apoptosis, ${ }^{17}$ it is therefore conceivable to hypothesize that myostatin exerts functional roles in regulating cancer cell growth or death by regulating energy metabolism. This is important since rapidly growing tumor

\footnotetext{
${ }^{1}$ The State Key Laboratory of Biomembrane and Membrane Biotechnology, Chinese Academy of Sciences, Beijing, China; ${ }^{2}$ The Graduate School of Chinese Academy of Sciences, Beijing, China; ${ }^{3}$ The National Laboratory of Medical Molecular Biology, Institute of Basic Sciences, Chinese Academy of Medical Sciences and School of Basic Medicine, Peking Union Medical College, Beijing, China and ${ }^{4}$ College of Life Sciences, Nankai University, Tianjin, China

${ }^{*}$ Corresponding author: Q Chen, The State Key Laboratory of Biomembrane and Membrane Biotechnology, Chinese Academy of Sciences, Beijing, China. Tel: + 861064807321 ; Fax: + 861064807321 ; E-mail: chenq@ioz.ac.cn

Or D Zhu, National Laboratory of Medical Molecular Biology, Institute of Basic Medical Sciences, Chinese Academy of Medical Sciences, School of Basic Medicine, 5 Dong Dan San Tiao, Beijing 100005, China. Tel: + 86106910 6949; Fax: + 86106510 5083; E-mail: dhzhu@ pumc.edu.cn or dhzhusara@gmail.com

${ }^{5}$ These authors contributed equally to this work.

Keywords: myostatin; apoptosis; VDAC1/HKIl; mitochondrial metabolism

Abbreviations: TGF- $\beta$, transforming growth factor- $\beta$; VDAC1, voltage-dependent anion channel 1; HKII, hexokinase II; ACTRII, activin receptor II; ALK, activin receptor-like kinase; OXPHOS, oxidative phosphorylation; ECAR, extracellular acidic ratio; OCR, oxygen consumption ratio; $\triangle \Psi \mathrm{\Psi m}$, membrane potential; TUNEL, terminal UTP nick end labeling; G-6-P, glucose 6-phosphate; 2DG, 2-deoxy-glucose; IN, inosine; SP, sodium pyruvate; MOMP, mitochondrial outer membrane permeabilization; $\mathrm{mPTP}$, mitochondrial permeability transition pore; CsA, Cyclosporin-A; GSK3 $\beta$, glucose synthase kinase $3 \beta$; PKB/Akt, protein kinase $B$ Received 10.7.12; revised 04.12.12; accepted 16.1.13; Edited by C Munoz-Pinedo
} 
cells typically display altered aerobic glycolysis (Warburg effect $)^{18}$ and metabolic dysregulation is related to tumor growth and cell death. ${ }^{19}$ In this report, we therefore tested this hypothesis and our results demonstrated that myostatin induces metabolic shift from oxidative phosphorylation (OXPHOS) to glycolysis in cancer cells and interestingly the chronic exposure of myostatin results in the activation of mitochondria-dependent apoptosis. In an effort to understand the underlying mechanism, we showed that upregulation of VDAC1 and Bax translocation to the mitochondria played critical role in myostatin-induced apoptosis in cancer cells. The findings presented in this study suggest that a better understanding of the mechanistic links between cancerous metabolism and growth control by myostatin may be useful for developing better treatments of human cancer.

\section{Results}

Myostatin induces mitochondrial metabolic alterations in cancer cells. To test the possibility that myostatin regulates mitochondrial metabolic activities in cancer cells, we first examined glucose consumption and lactate production in HeLa cells following treatment with myostatin. Glucose consumption (Figure 1a) and lactic acid production (Figure 1b) were significantly accelerated as early as 6 or $12 \mathrm{~h}$, respectively. A similar lactic acid production profile were also observed in various cancer cell lines including AZGY83A cell (a lung adenocarcinoma cell line) and MCF-7 cell (a breast cancer cell line), but these changes were less drastic in SW480 (a colorectal cancer cell line) and SH-SY5Y (a neuroblastoma cell line) (Figure 1c), indicating the differential function of myostatin in regulating mitochondrial metabolism in cancer cells. In support of this observation, we also examined the metabolic fluxes of both glycolysis and OXPHOS, and not surprisingly, observed a significant increase of ECAR (extracellular acidic ratio; an indicator of glycolysis flux) whereas a robust decline of OCR (oxygen consumption ratio; an indicator of OXPHOS) from 12 to $24 \mathrm{~h}$ (Figures 1d and e), which is supported by the observation that mstn-null mice showed slightly higher level of respiration ratio than their wild type littermates (Supplementary Figure S1). To further verify the effect of myostatin on mitochondrial metabolism, we assayed ATP concentration and found that the ATP level was maintained at early time points following treatment with myostatin, and that prolonged treatment caused drastic ATP reduction as early as $12 \mathrm{~h}$ and almost complete depletion by $36 \mathrm{~h}$ (Figure 1f). In addition, the loss of mitochondrial membrane potential $(\Delta \Psi \mathrm{m})$ became evident $24 \mathrm{~h}$ after myostatin treatment as measured by DioC $_{6},{ }^{3}$ a commonly used dye to measure mitochondrial membrane potential (Figure 1g; Supplementary Figure S2). Collectively, these results demonstrate that myostatin alters mitochondrial energy metabolism by switching glucose metabolism from OXPHOS to glycolysis in glucose-addicted cancer cells.

\footnotetext{
Myostatin induces apoptosis via mitochondrial apoptotic pathway in HeLa cells. We observed that prolonged exposure of myostatin caused significant loss of viability of the cell. We thus tested if myostatin induced the loss of
}

viability through a mechanism of apoptosis in HeLa cells. Annexin V-propidium iodate double staining clearly showed that myostatin induced phosphatidylserine externalization, a hallmark of apoptosis in a time- and dose-dependent manner (Figures $2 \mathrm{a}$ and b). Western blotting revealed a decrease in both procaspase- 9 and procaspase-3 levels following treatment with myostatin for $24 \mathrm{~h}$ (Figure 2c). The cleavage product of PARP, a substrate of caspase-3, was evident after $24 \mathrm{~h}$ of treatment (Figure 2c). We also measured the caspase activity in individual HeLa cells by FITC-VAD-FMK, a fluorescent marker specific for activated caspases. Caspases are activated after $24 \mathrm{~h}$ of myostatin treatment and can be potently inhibited by z-VAD, a pan-caspase inhibitor (Figure 2d). Furthermore, the presence of myostatin significantly reduced clonogenicity (Figure 2e), which further supports that myostatin induced apoptosis in HeLa cells. These results demonstrate that myostatin induced typical apoptosis, as characterized by the activation of caspase and the loss of clonogenicity.

Myostatin induced drastic mitochondrial morphological changes from a filamentous shape into a donut shape (Supplementary Figure S3). Similar changes were observed in cells under metabolic stress-induced cell death. ${ }^{20}$ To assert that myostatin induces mitochondrial-dependent apoptosis, we assessed whether cytochrome $c$ was redistributed from the mitochondria into the cytosol, where it activates caspase-9. Cytochrome c levels were significantly reduced in mitochondrial fractions, while were increased in cytosolic fractions after $24 \mathrm{~h}$ of treatment with myostatin, although the total cytochrome $c$ level remained unchanged (Figures $2 \mathrm{f}$ and $\mathrm{g}$ ). These results therefore showed that the mitochondrial pathway is involved in myostatin-induced apoptosis.

Myostatin inhibits tumor growth in xenograft tumor models. We next examined whether myostatin induces tumor cell apoptosis in xenograft tumor models. We first test the effect of myostatin on xenograft tumors in immunedeficient nude mice. Treatment with varying concentrations of myostatin did not affect the body weight of the animals treated (data not shown). Notably, we observed substantial reduction of tumor weight in the mice treated with myostatin (Figures $3 a$ and $b$ ). The average tumor size in the myostatintreated group was significantly reduced, compared with that of the vehicle group (Figure 3c). As a matter of fact, after 6 days of treatment with myostatin, 2 of the 10 treated mice achieved tumor regression below palpable levels. We then asked whether the reduced tumor size was due to tumor cells undergoing apoptosis as observed in in vitro cultured cancer cells. Indeed, TUNEL-staining analysis revealed a markedly increased number of apoptotic tumor cells in the myostatintreated tumor compared with the apoptotic cells from vehicle (PBS)-treated tumors (Figures $3 d$ and e; Supplementary Figure S4). Furthermore, functional role of myostatin in inhibiting tumor growth was also corroborated by approaching this question with an immune-competent xenograft mouse model. B16F10 cells, which are sensitive to myostatin-induced apoptosis (Supplementary Figure S5), were used to establish xenograft melanoma in $\mathrm{C} 57 \mathrm{BL} / 6$ mice. Treatment with myostatin significantly inhibited tumorigenesis and caused tumor cells apoptosis (Figures $3 f$ and $\mathrm{g}$ ), which is 
a

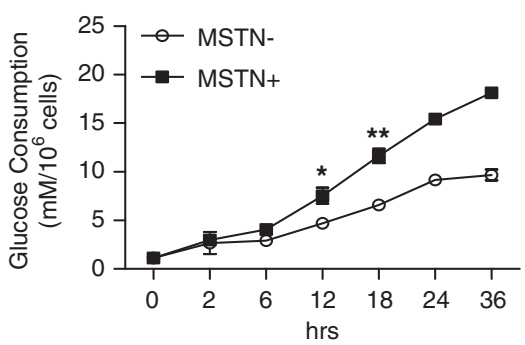

b

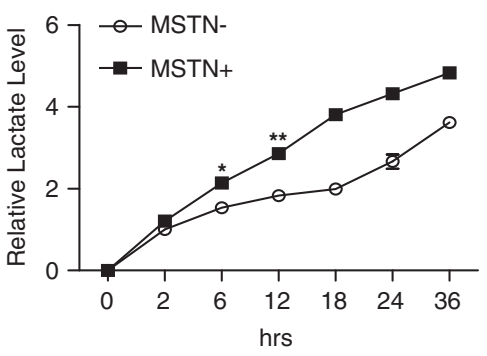

C

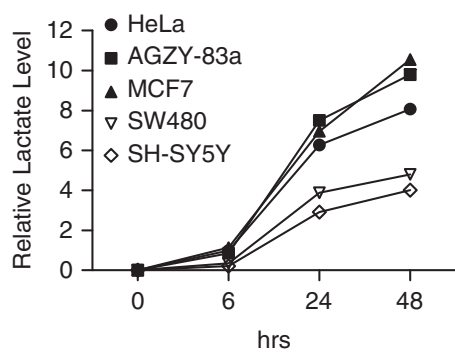

d $\quad 1.2$ Glucose Oligomycin 2-DG

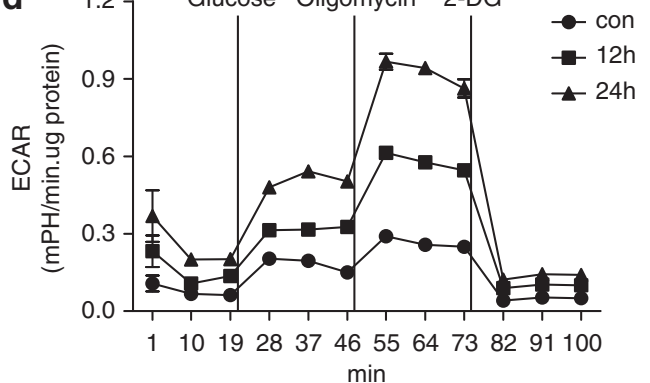

e
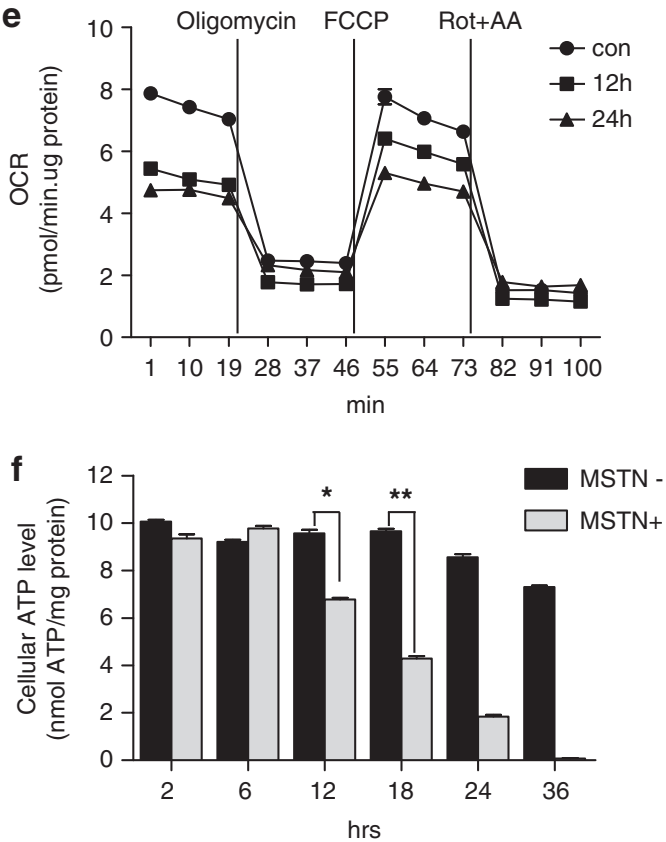

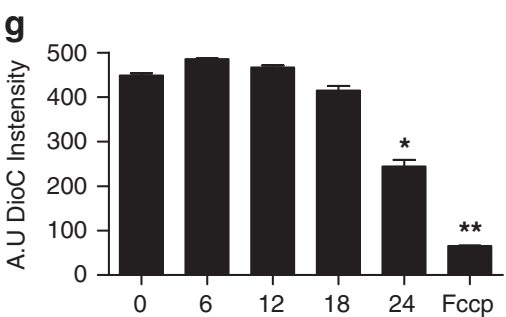

Figure 1 Myostatin induces metabolic alterations in HeLa cells. (a, b) Effect of myostatin on glucose consumption and lactate production. HeLa cells were treated with myostatin (if not specified, at a concentration of $500 \mathrm{ng} / \mathrm{ml})^{8}$ or PBS for the indicated times. (a) Glucose consumption and (b) Lactate production were monitored. (c) Relative lactate level in HeLa, AGZY-83a, MCF-7, SW480 and SH-SY5Y cells cultured in normal DMEM. (d, e) A series of ECAR and OCR in HeLa cells in the presence or absence of myostatin were examined by Seahorse XF24 analyzer. (d) ECAR following the addition of glucose defines glycolysis and ECAR following oligomycin represents maximum glycolytic capacity. ECAR following treatment with 2-DG represents acidification associated with non-glycolytic activity. Cells are subsequently treated with $4.5 \mathrm{~g} / \mathrm{l} \mathrm{D}-\mathrm{glucose}$, $1 \mu \mathrm{M}$ oligomycin and $100 \mathrm{mM}$ 2-deoxyglucose (2-DG). (e) Effect of myostatin on oxygen consumption. OCR prior to the addition of oligomycin (1 $\mu \mathrm{M})$ and following the treatment of $\mathrm{FCCP}(0.5 \mu \mathrm{M})$ represent the basal mitochondrial respiration and maximal mitochondrial respiration capacity, respectively. OCR following Rotenone (1 $\mu \mathrm{M})$ plus Antimycin- $\mathrm{A}(2 \mu \mathrm{M})$ treatment represents non-mitochondrial respiration. (f) Cellular ATP levels in the presence or absence of myostatin. Data are shown as mean values \pm S.E.M., $n=3$. (g) Mitochondrial membrane potential $(\Delta \Psi \mathrm{m})$ was analyzed by flow cytometry with $\mathrm{DiOC}_{6}{ }^{3}$ staining $(10 \mathrm{nM})$. FCCP $(2 \mu \mathrm{M})$ was used as a positive control of $\Delta \Psi \mathrm{m}$ loss

indicated by a much larger total area of cells with condensed nuclei (Figure 3h). Together, our results demonstrated that myostatin could induce not only cancer cells apoptosis in vitro, but also inhibit tumorigenesis in vivo in both immune-deficient and immune-competent xenograft tumor models.
Upregulation of VDAC1 is important for myostatininduced apoptosis. VDAC has been recognized as a key protein in mitochondria-mediated apoptosis and participates in mitochondrial metabolism. ${ }^{21-24}$ Therefore, it is conceivable to test the role of VDAC1 in myostatin-induced apoptosis. To this end, we first examined several cancer cell lines for 

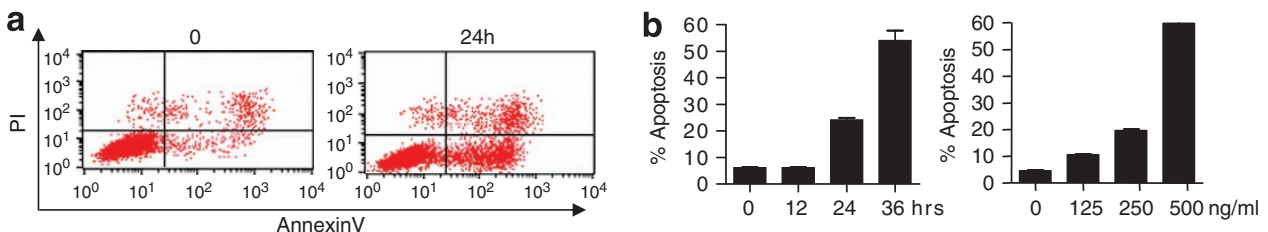

C

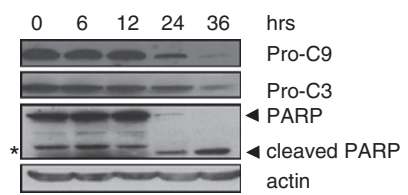

d
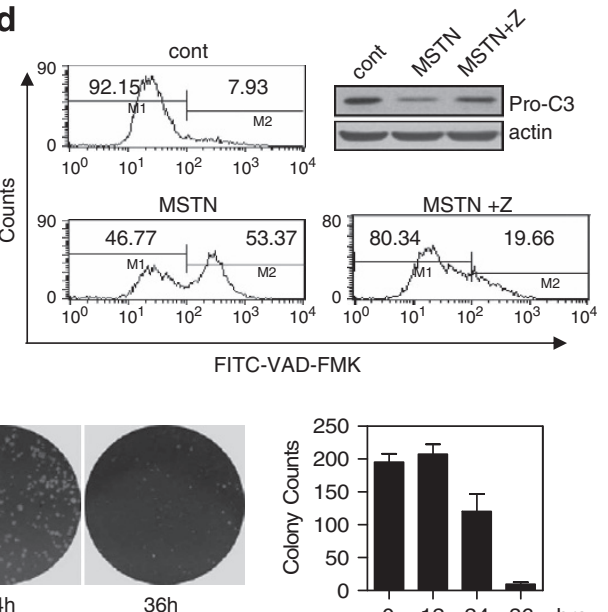

e
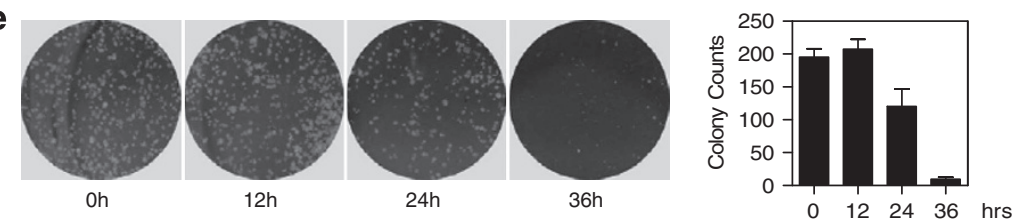

f
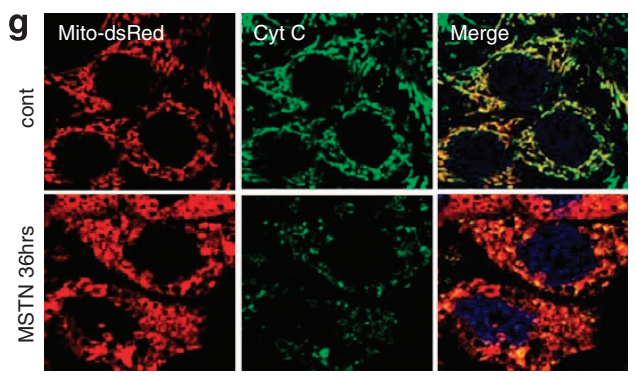

Figure 2 Myostatin induces mitochondrial apoptosis in HeLa cells. HeLa cells were treated with myostatin or PBS for the indicated times. (a) Annexin V/PI analysis following myostatin treatment. PI, propidium iodide. (b) Time course and dose response of HeLa cells in response to myostatin treatment. The percentage of apoptotic cells was detected with annexin V and PI double staining. Data are expressed as mean values \pm S.E.M., $n=3$. (c) The expression of caspase-9, caspase-3 and PARP were examined. Pro-C9, procaspase-9; Pro-C3, procaspase-3, *: non-specific signal. (d) Pan-caspase activity was analyzed using flow cytometry. (e) Clonogenicity of HeLa cells in the presence of myostatin. $(\mathbf{f}, \mathbf{g})$ Subcellular localization of cytochrome $\mathrm{C}$ was examined by western blotting (f) and immunostaining $(\mathbf{g})$

VDAC1 expression and sensitivity to myostatin. VDAC1 expression was markedly induced by myostatin in HeLa, AGZY-83a and MCF-7 cells, but remained unchanged in SW480 and SH-SY5Y cells (Figure 4a). Interestingly, we found that the AGZY-83a and MCF-7 cells, in which VDAC1 was upregulated by myostatin were also sensitive to myostatin-induced apoptosis, whereas the cancer cells in which VDAC1 expression was not induced either responded to only a limited extent (SW480), or showed complete resistance (SH-SY5Y) to myostatin treatment (Figure $4 \mathrm{~b}$ ). Notably, VDAC1 induction level and the sensitivity of different cancer cell lines to myostatin is positively correlated with their glycolytic activities (Figure 1c, measured as lactate production). These data indicate that VDAC1 induction is highly correlated with apoptosis induced by myostatin in those cancer cell lines addicted to glycolysis, suggesting a connection among glucose metabolism, VDAC1 induction and cancer cell apoptosis in response to myostatin.
To determine if the induction of VDAC1 is of functional importance for myostatin-induced apoptosis, VDAC1 was stably knocked down in HeLa cells. As shown in Figure 4c, myostatin-induced apoptosis was substantially attenuated in VDAC1-knockdown strains, $m 1$ and $m 2$. FITC-VAD-FMK staining showed significant impairment of myostatin-induced caspase activation in cells transfected with VDAC1-shRNA (Figure 4d). Together, our data strongly indicate that VDAC1 plays a critical role in mediating myostatin-induced apoptosis of cancer cells.

The first rate-limiting step in glucose metabolism is the conversion of glucose to glucose 6-phosphate (G-6-P) catalyzed by hexokinase, which is a key mediator of aerobic glycolysis. Hexokinase is known to directly couple intramitochondrial ATP synthesis to glucose metabolism and to bind to VDAC1 and inhibit apoptosis. ${ }^{25,26}$ We then examined the expression of Hexokinase II (HKII) and revealed that HKII protein level was decreased in HeLa cells treated with 
a

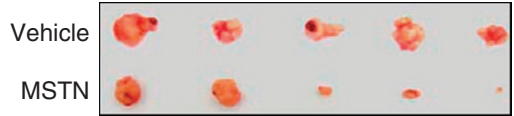

b

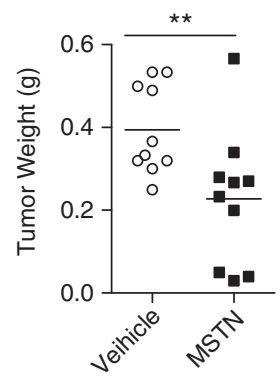

C

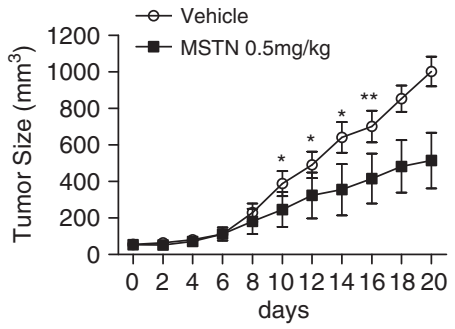

d

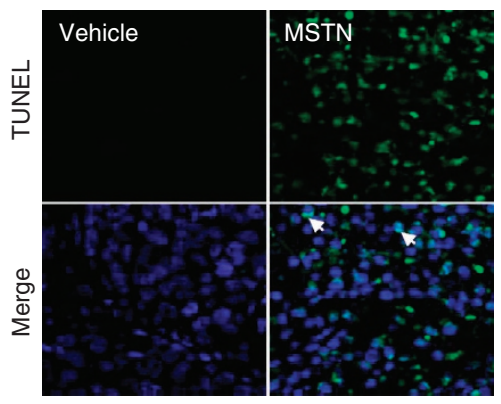

e
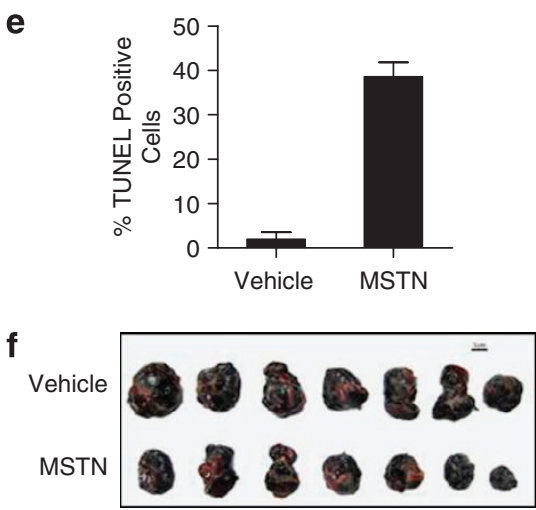

g

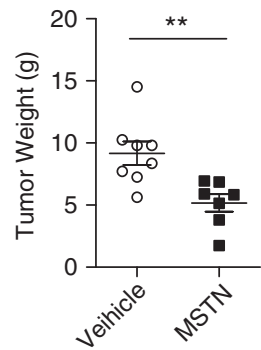

h

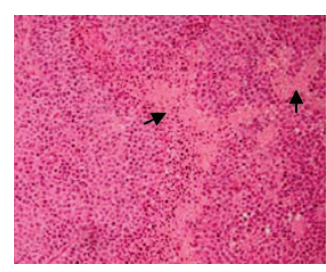

Vehicle

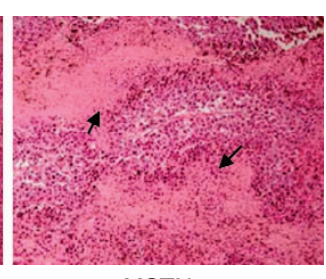

MSTN

Figure 3 Myostatin inhibits tumor growth in xenograft tumor model. (a-e) HeLa cells were injected into nude mice and myostatin were injected intratumorally. (a) Five representative xenograft tumors in each group with the treatment of vehicle or myostatin are shown. (b) Tumors weight was measured and shown in the scatter plot $(n=10)$. $P=0.0073$. (c) Tumor sizes were measured, and tumor volumes calculated ( $n=10$ per group). (d) Representative images of the fluorescent TUNEL staining of xenograft tumor sections. Arrowheads indicate apoptotic cells. Green: TUNEL-positive. (e) The percentages of apoptotic cells was quantified, and results are presented as mean values \pm S.E.M. of 10 sections. At least 200 cells from each section were counted. (f-h) B16F10 cells were injected into C57BL/6 mice and myostatin were injected intratumorally every other day. (f) Seven representative xenograft tumors in each group with the treatment of vehicle or myostatin are shown. (g) Tumors weight was measured and shown in the scatter plot $(n=13) . P=0.0055$. (h) Representative images of the hematoxylin and eosin staining of xenograft tumor sections. Arrowheads indicate the regions enriched with apoptotic cells, indicated by condensed nuclei

myostatin in a time-dependent manner (Figure 4e). Fractionation analysis further showed that mitochondrial HKII, consistent with the pattern of total HKII, decreased at $24 \mathrm{~h}$ treatment (Figure 4f). This is in contrast to the expression level of VDAC1, which was found to be highly induced in the presence of myostatin in a time-dependent manner (Figures $4 a$, e and $\mathrm{f}$ ). However, other mitochondrial markers, including Tim23 and UCP2 for inner membrane, and cyclophilin D and MnSOD for matrix proteins remained unchanged. To further explore whether HKII is involved in protecting cells from myostatininduced apoptosis, HeLa cells with stably overexpressed HKII were treated with myostatin, and both Annexin V/PI staining and FITC-VAD-FMK showed a significant reduction of apoptosis in those cells with overexpressed HKII (Figures $4 \mathrm{~g}$ and $\mathrm{h}$ ). In addition, we were able to show myostatininduced expression of VDAC1 was due to neither transcriptional regulation nor the increased de novo protein synthesis (Supplementary Figure S6). Taken together, our data revealed that the observed changes in HKII and VDAC protein levels are myostatin-specific responses, and that these changes are closely linked with myostatin-induced apoptosis in cancer cells.

ATP depletion is responsible for myostatin-induced VDAC1 upregulation and apoptosis. We next are prompted to ask if metabolic changes and ATP depletion is causally linked with mitochondrial-dependent apoptosis in cancer cells. To test this, we used 2-deoxy-glucose (2DG) to mimic ATP depletion (Figure 5a) through glucose deprivation. Western blotting showed that 2DG alone was sufficient to induce VDAC1 expression (Figure 5b). HeLa cells were then treated with myostatin in the presence of 2DG and we 
a

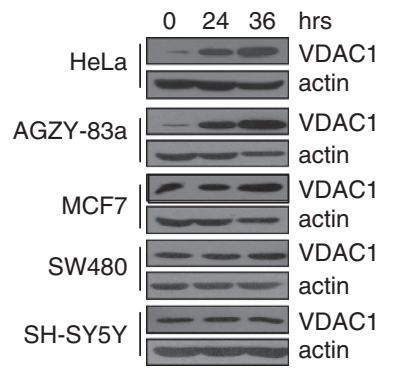

C

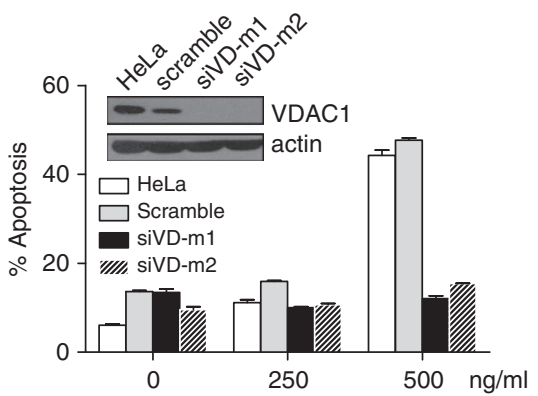

b

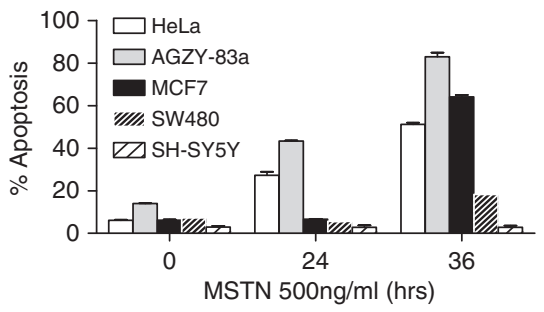

d

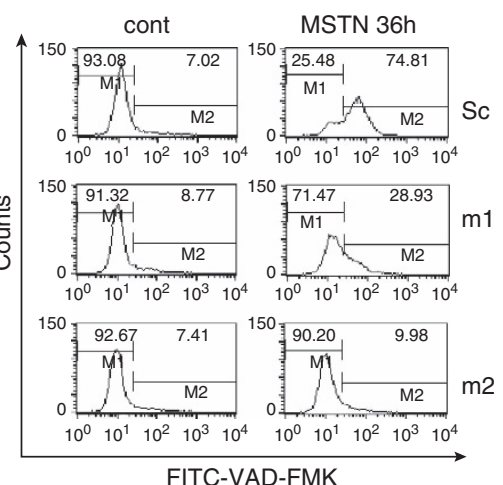

e

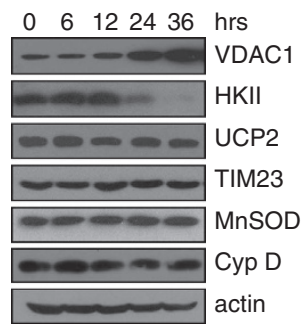

g

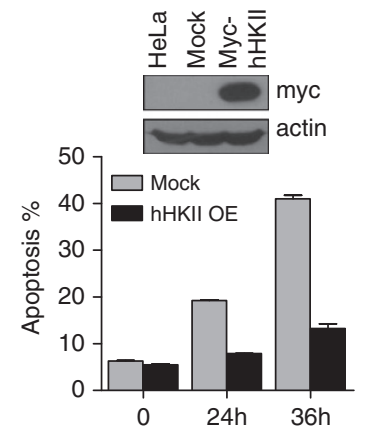

f

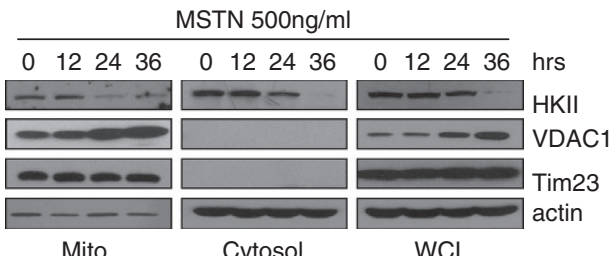

h

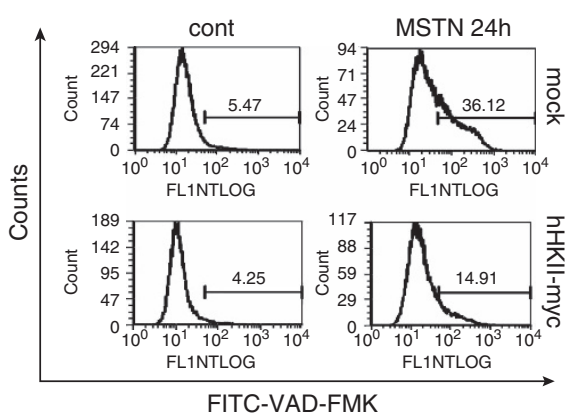

Figure 4 VDAC1 is important in myostatin-induced apoptosis. (a) Expression of VDAC1 in different cell lines with the treatment of myostatin for the indicated times. (b) Different cancer cell lines demonstrate varied sensitivity to myostatin treatment. Apoptosis was analyzed by Annexin V/PI staining. (c, d) HeLa cells stably transfected with scrambled or VDAC1 shRNA were treated with myostatin or PBS for $36 \mathrm{~h}$ at the indicated concentrations. siVD-m1: stable clone 1; siVD-m2: stable clone 2. Apoptosis (c) and pan-caspase activity (d) were analyzed using flow cytometry. (e) HeLa cells were treated with myostatin and cell lysates were analyzed by western blotting with antibodies for VDAC1, HKII, Cyclophilin D, UCP2, Tim and MnSOD. (f) Localization of HKII, VDAC1 and Tim in different fractionations of HeLa cells with the treatment of myostatin. $(\mathbf{g}, \mathbf{h})$ HeLa cells, stably transfected with myc-HKII (upper panel of $\mathbf{g}$ ), were treated with PBS or myostatin for the time as indicated. Apoptosis (lower panel of $\mathbf{g}$ ) and pancaspase activity (h) were analyzed using flow cytometry

found that 2DG significantly enhanced myostatin-induced apoptosis of the HeLa cells (Figure 5c). Mitochondrial respiratory inhibitors such as rotenone (complex I), antimycin A (complex III) and oligomycin (complex V) inhibited OXPHOS resulting in ATP depletion, and interestingly, they all increased VDAC protein expression (Supplementary
Figure S7). The effects of mitochondrial inhibitors further support the observation that VDAC1 can be induced in response to ATP depletion and consequentially lead to apoptosis. Interestingly, inosine (IN) and sodium pyruvate (SP), which are routinely used to restore cellular ATP levels, ${ }^{27,28}$ rescue ATP depletion and partially inhibited 

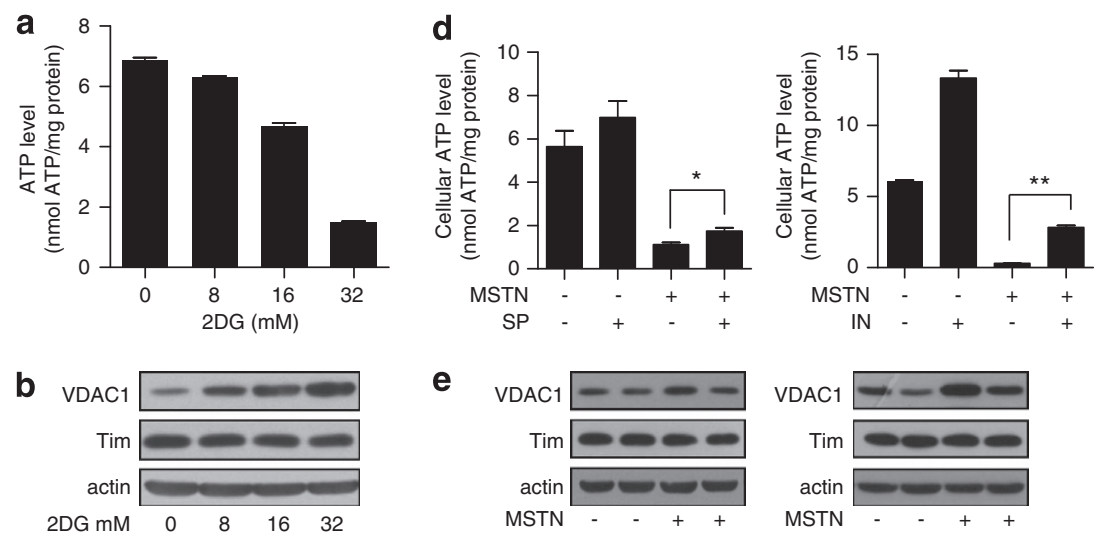

e
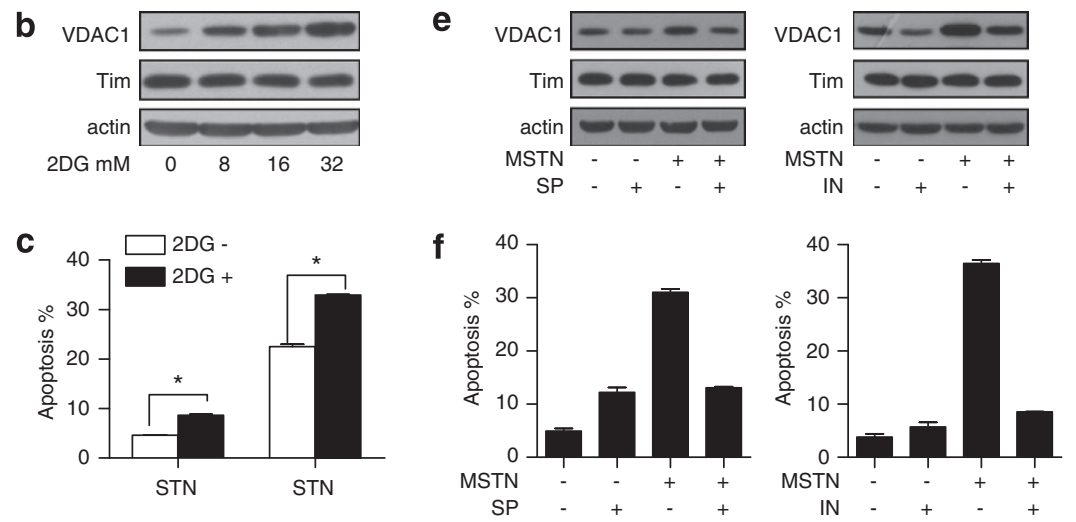

Figure 5 ATP depletion mediates myostatin-induced VDAC1 upregulation, which contributes to apoptosis. (a, b) HeLa cells cultured in low glucose (5.5 mM) DMEM were treated with vehicle, $8 \mathrm{mM}, 16 \mathrm{mM}$ or $32 \mathrm{mM} 2 \mathrm{DG}$ for $24 \mathrm{~h}$. Cells were harvested for the ATP assay (a) and western blotting analysis (b). (c) HeLa cells pre-incubated with or without 2DG (32 mM) for 30 min were treated with PBS or myostatin for $24 \mathrm{~h}$, followed by Annexin V/PI staining. (d) HeLa cells were incubated in glucose-free DMEM in the presence or absence of sodium pyruvate $(10 \mathrm{mM})$ or inosine $(20 \mathrm{mM})$, followed by treatment with myostatin $(500 \mathrm{ng} / \mathrm{ml})$ for $6 \mathrm{~h}$. Cellular ATP levels were examined $(n=3)$. (e) HeLa cells were treated as in (d), and cells lysates were subjected to western blotting analysis with anti-VDAC1 antibody. (f) HeLa cells were treated as in (d) with extended myostatin treatment for $12 \mathrm{~h}$, followed by Annexin $\mathrm{V}$ and PI double staining

myostatin-induced VDAC1 upregulation (Figure 5e) and apoptosis (Figure $5 \mathrm{f}$ ). Combined, all of these results support the conclusion that ATP depletion is responsible for myostatin-induced VDAC1 upregulation and apoptosis.

Mitochondrial translocation of Bax is involved in myostatin-induced apoptosis. Previous studies have shown that detachment of HK II from the mitochondria promoted the translocation of Bax to mitochondria for subsequent cytochrome c release. ${ }^{25,29,30}$ Consistent with this, we found that myostatin treatment causes Bax translocation to mitochondria as revealed by immunostaining of Bax protein (Figure 6a). This observation was further confirmed by fractionation analysis as shown in Figure 6b. In addition, the expression profile of $\mathrm{Bcl}-2$ family members following myostatin treatment clearly showed that the antiapoptotic $\mathrm{Bcl}-2$ family proteins $\mathrm{Bcl}-2, \mathrm{Mcl}-1$ remain unaltered (Figure 6c). The protein levels of the pro-apoptotic proteins of Bax and Bak are also not changed, while Bim and Bik levels were reduced following myostatin treatment (Figure 6c). Further analysis shows that myostatin-induced apoptosis is largely inhibited in the Bax-deficient HCT116 cells (Supplementary Figure S8A), indicating the specific role of Bax in myostatin-induced apoptosis. Although we did not detect direct interaction between Bax and VDAC1 (likely because Bax:VDAC1 interaction is transient and weak), we observed significant inhibition of Bax translocation and cytochrome $c$ release when VDAC1 was silenced with shRNA (Figures 6d and e). As expected, overexpression of
$\mathrm{Bcl}-2$ also prevents myostatin-induced Bax translocation and apoptosis (Supplementary Figures S8B-D). We thus conclude that chronic exposure of myostatin causes metabolic stresses leading to VDAC1 upregulation, Bax translocation to mitochondria and subsequent apoptosis in cancer cells.

\section{Discussion}

Within the present study we have provided compelling evidence that myostatin shifts glucose metabolism towards glycolysis and induces apoptosis in cancer cells. Exposure to myostatin resulted in mitochondrial morphological, physiological and functional changes, leading to the mitochondriadependent apoptosis in cancer cells (Figure 6f). We found that myostatin affects the protein levels of HK II and VDAC1, two key regulators of glucose metabolisms. The alteration of mitochondrial metabolism could lead to dissociation of HKII from the mitochondria and Bax translocation to the mitochondria, resulting in the metabolic stress-induced apoptosis.

Although the importance of activin/TGF- $\beta$ and their receptors on apoptosis and cancer biology is well recognized, to our best knowledge, our work represents the first report showing that myostatin induces mitochondria-dependent apoptosis in cancer cells. TGF- $\beta$ and related family members may transcriptionally regulate apoptosis-related genes, such as $\mathrm{XIAP}, \mathrm{Bcl}-2 / \mathrm{Bcl}-\mathrm{xl}$ or Bim. Alternatively, they regulate apoptosis through non-canonical pathways, such as JNK and Akt signaling, leading to the phosphorylation and activation of the pro-apoptotic molecules. ${ }^{31,32}$ Nonetheless, upon myostatin 

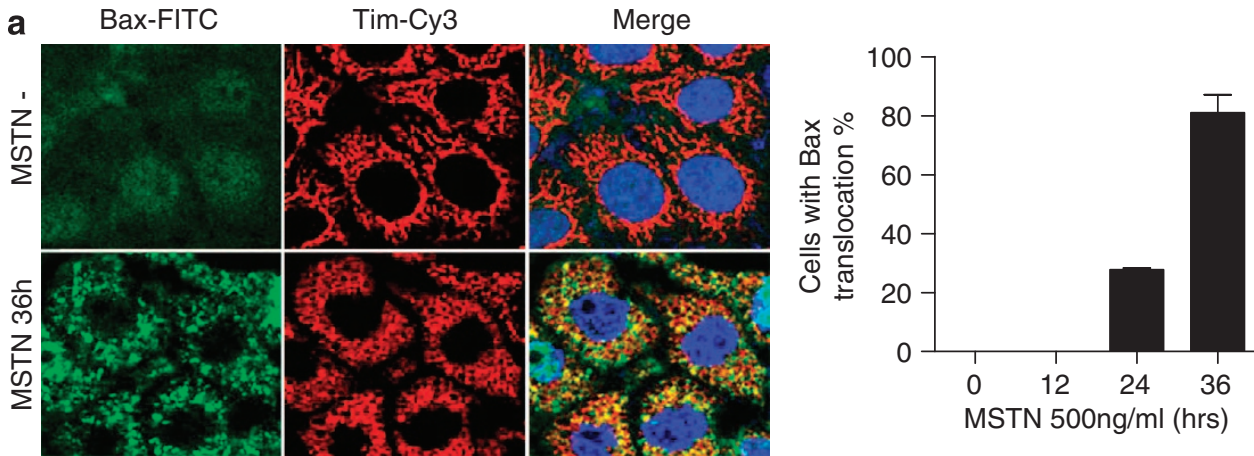

b

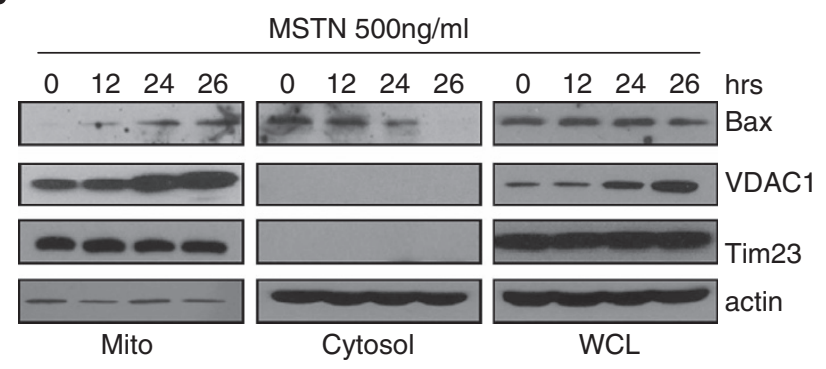

C

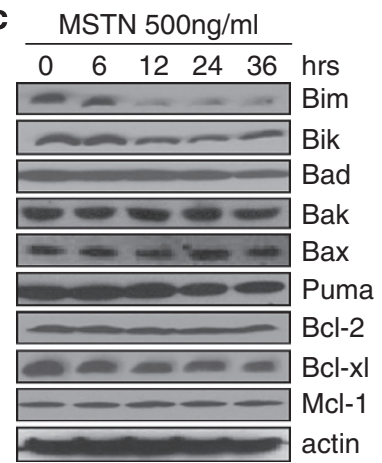

d

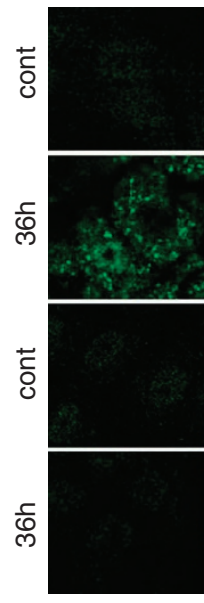

Bax

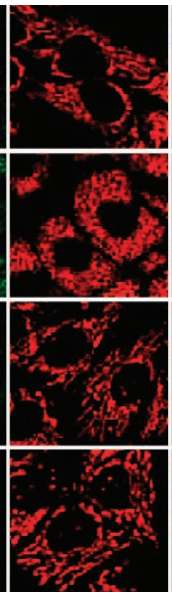

Tim

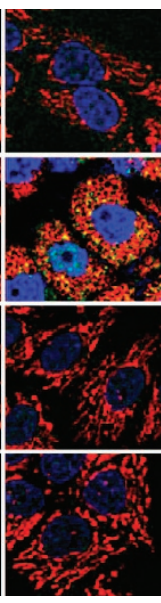

Merge

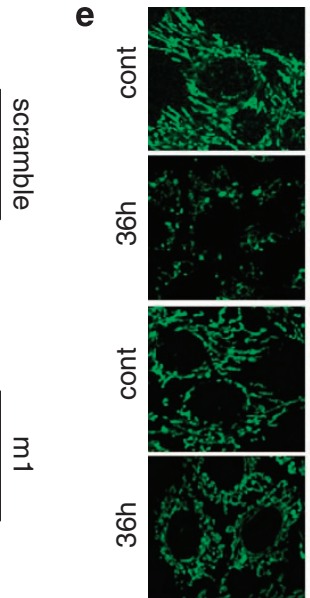

CytC

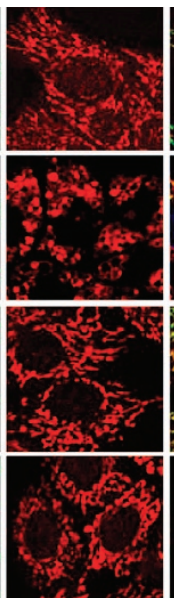

Tim

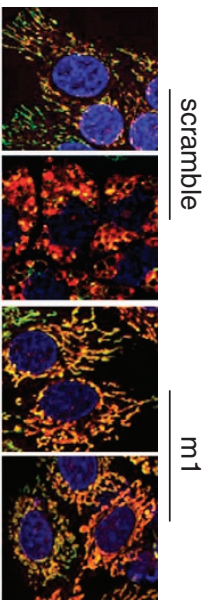

Merge

f

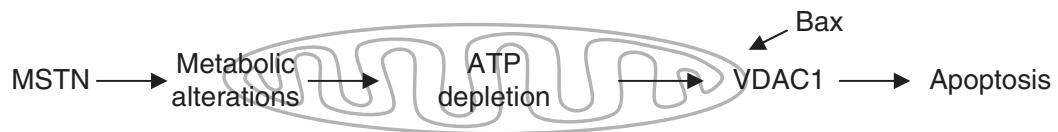

Figure 6 Mitochondrial translocation of Bax is involved in VDAC1-mediated apoptosis, which is induced by myostatin. (a) Left panel: HeLa cells treated with myostatin for $36 \mathrm{~h}$ were fixed and stained with anti-BAX (green) and anti-TIM (red) antibodies. Right panel: Statistics of HeLa cells with redistributed Bax in the presence of myostatin for $36 \mathrm{~h}$. The data were shown as mean \pm S.E.M. (b) Subcellular localization of VDAC1 and Bax were examined by western blotting. Tim was shown as a loading control of mitochondrial sample; actin was shown as an loading control of cytosolic fraction and whole cell lysate. (c) Expression profile of major members of Bcl-2 family. (d) Scramble and siVD-m1 cells were treated with PBS or myostatin for $36 \mathrm{~h}$. Immunofluorescence was performed with antibodies for Bax (green) and Tim (red). (e) HeLa cells were treated as in (d). Green: Cytochrome C; Red: Tim-Cy3. (f) A model for the mechanism underlying myostatin induction of VDAC1-mediated apoptosis in cancer cells

treatment, we did not find any induction or phosphorylation of pro-apoptotic $\mathrm{Bcl}-2$ family proteins, such as Bim and Bad, nor did we see the drastic changes of anti-apoptotic Bcl-2 molecules. Although $\mathrm{Bcl}-\mathrm{xl}$ was slightly decreased in response to myostatin, the functional analysis did not show any correlation with myostatin-induced apoptosis. Instead, we clearly showed that myostatin activates Bax-dependent apoptosis. Bax translocation to the mitochondria upon myostatin treatment is highly correlated with the onset of apoptosis and cytochrome $\mathrm{c}$ release. 
It is recognized that dissociation of hexokinase from mitochondrial VDAC regulates Bax translocation to the mitochondria. ${ }^{25,29,30,33}$ Our finding is the first to suggest that such a mechanism is likely involved in myostatin-induced apoptosis. We demonstrated that VDAC1 plays a critical role in myostatin-induced apoptosis of cancer cells (Figures 4 and 5). One theory, albeit controversial, ${ }^{34}$ is that closure of the VDAC 1 channel prevents efficient exchange of ATP and ADP between the cytosol and mitochondrial matrix, leading to mitochondria swelling and outer membrane permeabilization (MOMP), and thereby release of pro-apoptotic factors from the intermembrane space..$^{21,22,35,36}$ Indeed, we observed a loss of mitochondrial membrane potential, indicative of the opening of mitochondrial permeability transition pore (mPTP), during the onset of myostatin-induced apoptosis. Interestingly, we also found that Cyclosporin-A (CsA), a selective inhibitor of mPTP (Supplementary Figure S9), can compromise the apoptosis-inducing function of myostatin, implying that MPTP may also contribute to myostatin-induced apoptosis. Alternatively, VDAC could interact with $\mathrm{HKII}$ or $\mathrm{Bcl}-2$ family proteins, thus regulating apoptosis. ${ }^{37,38}$ Indeed, we observed that dissociation of HKII from the mitochondria occurs, concomitantly, with the translocation of Bax to the mitochondria. Akt and GSK3 $\beta$ are implicated in regulating the association of HKII with VDAC1. ${ }^{30,38,39}$ Interestingly, we previously reported that myostatin could activate GSK3 $\beta$ and induce cell-cycle arrest through the inhibition of Akt in the myoblast, ${ }^{8}$ which aids in understanding the case in cancer cells, in which prolonged treatment of myostatin may not sustain Akt activity, and instead leads to the dissociation of HKII from mitochondria.

It is intriguing that the changes in VDAC and HKII protein levels are inversely correlated upon myostatin treatment. As was reported, depending on the cellular metabolic state, HKII may either promote or inhibit apoptosis. ${ }^{40}$ Interestingly, we found that perturbation of intracellular ATP levels, either by application of 2DG or inhibitors of respiratory chain, could induce VDAC1 protein levels as we observed in myostatintreated cancer cell, whereas replenishment of ATP prevented VDAC1 upregulation and myostatin-induced apoptosis. It is possible that, when ATP level are decreased, as a compensatory response the cell may enhance the stability of VDAC1 for better transportation of ATP to supply enough energy for cell survival. Apart from GSK3 $\beta$, it is known that a number of metabolism-related kinases, such as PKA and PKC phosphorylate VDAC1 and regulate the interaction between VDAC1 and Bcl-2 family proteins, which in turn regulates respiration and apoptosis. ${ }^{38,41}$ Additionally, it was known that phosphorylation of VDAC1 may trigger conformational changes of VDAC1 and inhibit its degradation, resulting in VDAC1 accumulation. ${ }^{25,42}$ Indeed, the real-time PCR results showed that myostatin did not alter the mRNA level of VDAC1, whereas induction of VDAC1 in response to myostatin treatment could not be blocked by inhibiting de novo protein synthesis with cycloheximide (Supplementary Figure S6D), which suggests that myostatin could possibly enhance VDAC1 stability. On the other hand, phosphorylation of VDAC1 resulted in the dissociation of HK from the mitochondria, ${ }^{39}$ thus reducing its stability in cytosol. Intriguingly, the total HKII activity maintained to the same level with untreated cells, even though we observed drastic decline of its expression at $24 \mathrm{~h}$ treatment (Supplementary Figure S10). While the exact mechanism of VDAC1 and HKII stability awaits further investigation, our data suggest that altered ATP levels and metabolic stress affect mitochondrial-dependent apoptosis.

Myostatin is regarded as a potential molecular target for a number of diseases, including muscular dystrophy, sarcopenia, cachexia and diabetes. ${ }^{43,44}$ Our results are the first to suggest that myostatin could be useful in fighting cancers that have altered glycolytic activity and VDAC1 response. Our results from both in vitro and xenograft tumor models further indicate that focal expression or intratumoral injection of myostatin may be effective ways to combat cancer, while avoiding deteriorating effects on muscle. Both HKII and VDAC1 could serve as targets for cancer therapy as previously suggested. ${ }^{36,45-47}$ As a matter of fact, myostatin is known to be secreted in a latent form and is maintained at a comparatively high concentration in muscle, where it tends to be activated under acidic and hypoxic conditions due to high levels of glycolysis. ${ }^{16,48}$ Therefore, it is not surprising that myostatin might have specific proapoptotic functions in tumor tissue, but not in normal tissue, because most tumor cells predominantly produce ATP through glycolysis, and thus form a heterogeneous acidic and hypoxic microenvironment, which is similar to the case of muscle after exercise. In order to testify this hypothesis, we designed a series examination on different steps of myostatin-induced apoptosis in different cancer cell lines. Surprisingly, the cell-type-dependent sensitivity to myostatin significantly correlates to their glycolytic activity ( $r=0.991, P=0.0005$ ) (Supplementary Figures S11A and B; Figure 1c), whereas shows no correlation with the basal expression level of ACTRIIB, VDAC1 (Supplementary Figures S11C-E), or the quiescent ATP level (Supplementary Figures $\mathrm{S} 11 \mathrm{H}$ and I) in different cells, although the decrease of ATP and the induction of VDAC1 have been shown to be necessary for myostatin-induced apoptosis. Moreover, Lactate accumulation in the medium could be influenced by several factors besides the real glycolysis level, such as the proliferation rates of different cancer cells. However, we do see the big variation among cells derived from different cancers, but they showed no correlation with myostatin susceptibility (Supplementary Figures S11F and G). Together, our results may help to understand, at least in part, why there is a low incidence of cancer in skeletal muscle, and how physical exercise could prevent cancer. During exercise, glucose is rapidly utilized by muscle and lactate is highly produced, as observed in our system. These findings may also aid in understanding why cells addicted to glucose are more sensitive to myostatin, and serve to explain why certain malignant tumor cells are more sensitive to myostatin than cells in normal tissue.

\section{Materials and Methods}

Cell culture and transfection. HeLa, AGZY-83a, MCF-7, SW480, SHSY5Y and B16F10 cells were grown in DMEM supplemented with $10 \%$ FBS (Hyclone, Logan, UT, USA) and $1 \%$ penicillin-streptomycin at $37^{\circ} \mathrm{C}$ under $5 \%$ $\mathrm{CO}_{2}$. The targeting sequence in VDAC1 for RNA interference, $5^{\prime}$ aaAGTGACGGGCAGTCTGGAA $3^{\prime}$, was reported earlier by Yuan et al. ${ }^{42}$ Primers for shRNA were designed according to the manufacturer's instructions (Ambion, Carlsbad, CA, USA), and cloned into psilencer2.1-neo vector. To establish stable 
cell lines, HeLa cells were transfected with sh-VDAC1 or pcDNA4TOB-myc-HKII, and screened with neomycin and ziocin, respectively. Recombinant myostatin was expressed and purified as described previously. ${ }^{49}$ Exponentially growing cells were subjected to various treatments.

Reagents and antibodies. Antibodies against PARP, cytochrome $c$ and Tim were obtained from BD Biosciences (San Jose, CA, USA). Caspase-9 and caspase-3 antibodies were acquired from Cell Signaling (Danvers, MA, USA), and the anti-VDAC1 monoclonal antibody from Abcam (Cambridge, MA, USA). SP, inosine and 2-deoxy-D-glucose were purchased from Sigma Chemical Co. (St. Louis, MO, USA). TMRM and $\mathrm{DiOC}_{6}{ }^{3}$ were acquired from Invitrogen (Carlsbad, CA, USA). The caspACE FITC-VAD-FMK in situ marker was purchased from Promega (Madison, WI, USA).

SDS-PAGE and western blot. Cells or membrane fractions were lysed in lysis buffer (10 mM HEPES, pH 7.4, $2 \mathrm{mM}$ EGTA, 0.5\% NP-40, protease inhibitors). Equivalent protein quantities $(20 \mu \mathrm{g})$ were subjected to SDS-PAGE, and transferred to nitrocellulose membranes. Membranes were probed with the indicated primary antibodies, followed by the appropriate HRP-conjugated secondary antibodies (KPL, Gaithersburg, MD, USA). Immunoreactive bands were visualized with a chemiluminescence kit (Pierce, Rockford, IL, USA).

Subcellular fractionation. Subcellular fractionation was performed as previously described. ${ }^{50}$ Cells were harvested and resuspended in hypotonic buffer. After gentle homogenization with a Dounce homogenizer, cell lysates were subjected to differential centrifugation. The resulting membrane fractions were lysed, and analyzed using western blot.

Immunofluorescence microscopy. Cells were grown to $60 \%$ confluence on a coverslip. After treatment, cells were washed twice with PBS, and fixed with freshly prepared $3.7 \%$ formaldehyde at $37^{\circ} \mathrm{C}$ for $15 \mathrm{~min}$. Antigen accessibility was increased by treatment with $0.2 \%$ Triton $\mathrm{X}-100$. Cells were incubated with primary antibody for $1 \mathrm{~h}$, and, after washing, stained with a secondary antibody for a further $45 \mathrm{~min}$. Cell images were captured with an LSM 510 Zeiss confocal microscope (Carl Zeiss, Jena, Germany). DAPI staining was used to identify nuclei.

Apoptosis assay. Apoptosis was assessed by detecting phosphatidylserine exposure on the cell membrane with Annexin V staining as described previously. ${ }^{51}$ In situ caspase activation was determined by detecting FITC-VAD-FMK fluorescence with flow cytometry as described previously.52 TUNEL analysis was performed with an in situ death detection kit (Roche Molecular Biochemicals, Mannheim, Germany) as described previously. ${ }^{52}$

Clonogenic assay. In brief, cells were pre-incubated for various periods of time in the presence of myostatin. Subsequently, cells with each treatment were plated in a six-well plate at 1000 cells per well in triplicates. Cell colonies were stained with crystal violet and then counted 10 days after initial plating.

\section{Mitochondrial membrane potential assay and metabolic events} measurements. Mitochondrial membrane potential was measured with $\mathrm{DiOC}_{6}{ }^{3}$ as described previously. ${ }^{36}$ Metabolic events were detected according to the protocols from Methods in Cell Biology. ${ }^{53}$ Intracellular ATP levels were determined using a commercially available colorimetric ATP assay kit (Biyuntian Co., Shanghai, China) according to the manufacturer's instructions. ATP levels were normalized to protein levels. Glucose consumption and the lactate level were evaluated with commercial kits (Biovision, Mountain View, CA, USA).

OCR and ECAR were evaluated by using a Seahorse XF24 Analyzer (Seahorse Bioscience, North Billerica, MA, USA) as described. ${ }^{54}$ In brief, $2 \times 10^{4}$ HeLa cells were seeded $24 \mathrm{~h}$ before myostatin treatment. The OCR is measured sequentially before or after the addition of oligomycin $(1 \mu \mathrm{M}), \operatorname{FCCP}(0.5 \mu \mathrm{M})$ and rotenone $(1 \mu \mathrm{M})$ plus antimycin-A $(2 \mu \mathrm{M})$. The ECAR is determined sequentially before or after the addition of D-glucose $(4.5 \mathrm{~g} / \mathrm{l})$, oligomycin $(1 \mu \mathrm{M})$ and 2-DG $(100 \mathrm{mM})$.

Tumor xenograft studies. Five-week-old female nu/nu nude mice were injected in the right posterior flank region with $2.5 \times 10^{6} \mathrm{HeLa}$ cells in $0.1 \mathrm{ml}$ of medium. Seven days after implantation, when most of the tumors had grown up to $75 \mathrm{~mm}^{3}$, animals were randomly selected to receive either vehicle $(n=10)$ or treatment $(n=10)$, followed by direct intratumoral injection of PBS or myostatin at a concentration of $0.5 \mathrm{mg} / \mathrm{kg} / \mathrm{day}$. Tumor sizes and body weights of mice were recorded every other day. Tumor dimensions were measured with calipers, and the volumes calculated using the following formula: volume $=0.5 \times I \times w^{2}$, with $I$ representing the maximal length and $w$ the width. After treatment for 3 weeks, all mice were sacrificed and carcinoma tissue evaluated using fluorescenceconjugated TUNEL assay.

For melanoma xenograft model, $1 \times 10^{6}$ B16F10 cells were injected subcutaneously into C57BL/6 mice. Ten days after implantation, when palpable tumor formed, myostatin were injected into the tumor mass at a dose of $0.5 \mathrm{mg} / \mathrm{kg}$ every other day. PBS-injected mice served as controls $(n=13)$. Tumors were measured every 2 days, and volume was calculated (length $\times$ width $^{2} \times 0.5$ ). All mice were sacrificed 21 days after cell injection and tumor tissue were collected and weighed. A 5- $\mu \mathrm{m}$ thick formalinfixed tumor sections were stained with hematoxylin and eosin.

Statistical analysis. In quantitative analyses using cultured cells represented as histograms, values were obtained from three independent experiments, and expressed as the mean \pm S.E.M. Statistical analysis was performed using the Student's $t$-test, with $P$-values $<0.05$ considered significant. ${ }^{*} P<0.05$ and ${ }^{*} P<0.01$ versus the corresponding controls are indicated. All statistical data were calculated with GraphPad Prism software (GraphPad Software, Inc., La Jolla, CA, USA).

\section{Conflict of Interest}

The authors declare no conflict of interest.

Acknowledgements. We thank Dr. Peter L Pedersen (John Hopkins University) and Dr. Liu Shusen (Institute of Zoology, CAS) for useful discussion, Laura Tollini (University of North Carolina at Chapel Hill) and Dr. Zhou Aimin (Cleveland State University) for editorial assistance. This work was supported by grants from the National Basic Research Program of China (Nos. 2011CBA01104, 2009CB521802, 2011CB910903), the National Natural Science Foundation of China (Nos. 31030041, 81130045 and 30671069).

1. McPherron AC, Lawler AM, Lee SJ. Regulation of skeletal muscle mass in mice by a new TGF-beta superfamily member. Nature 1997; 387: 83-90.

2. Lee SJ. Regulation of muscle mass by myostatin. Annu Rev Cell Dev Biol 2004; 20: 61-86.

3. Lee SJ. Sprinting without myostatin: a genetic determinant of athletic prowess. Trends Genet 2007; 23: 475-477.

4. Schuelke M, Wagner KR, Stolz LE, Hubner C, Riebel T, Komen W et al. Myostatin mutation associated with gross muscle hypertrophy in a child. N Engl J Med 2004; 350: 2682-2688.

5. Lee SJ, McPherron AC. Regulation of myostatin activity and muscle growth. Proc Natl Acad Sci USA 2001; 98: 9306-9311.

6. Kemaladewi DU, de Gorter DJ, Aartsma-Rus A, van Ommen GJ, ten Dijke P, t Hoen PA et al. Cell-type specific regulation of myostatin signaling. FASEB J 2011; 26: 1462-1472.

7. Wrana JL, Attisano L, Carcamo J, Zentella A, Doody J, Laiho M et al. TGF beta signals through a heteromeric protein kinase receptor complex. cell 1992; 71: 1003-1014.

8. Yang W, Zhang Y, Li Y, Wu Z, Zhu D. Myostatin induces cyclin D1 degradation to cause cell cycle arrest through a phosphatidylinositol 3-kinase/AKT/GSK-3 beta pathway and is antagonized by insulin-like growth factor 1. J Biol Chem 2007; 282: 3799-3808.

9. Morissette MR, Cook SA, Foo S, McKoy G, Ashida N, Novikov M et al. Myostatin regulates cardiomyocyte growth through modulation of Akt signaling. Circ Res 2006; 99: 15-24.

10. Chen $\mathrm{Y}, \mathrm{Ye} \mathrm{J}, \mathrm{Cao} \mathrm{L}$, Zhang Y, Xia W, Zhu D. Myostatin regulates glucose metabolism via the AMP-activated protein kinase pathway in skeletal muscle cells. Int J Biochem Cell Biol 2010; 42: 2072-2081.

11. Mitchell MD, Osepchook CC, Leung KC, McMahon CD, Bass JJ. Myostatin is a human placental product that regulates glucose uptake. J Clin Endocrinol Metab 2006; 91: 1434-1437.

12. McPherron AC, Lee SJ. Suppression of body fat accumulation in myostatin-deficient mice. J Clin Invest 2002; 109: 595-601.

13. Antony N, Bass JJ, McMahon CD, Mitchell MD. Myostatin regulates glucose uptake in BeWo cells. Am J Physiol Endocrinol Metab 2007; 293: E1296-E1302.

14. Guo T, Jou W, Chanturiya T, Portas J, Gavrilova O, McPherron AC. Myostatin inhibition in muscle, but not adipose tissue, decreases fat mass and improves insulin sensitivity. PLoS One 2009; 4: e4937.

15. Zhou X, Wang JL, Lu J, Song Y, Kwak KS, Jiao Q et al. Reversal of cancer cachexia and muscle wasting by ActRIIB antagonism leads to prolonged survival. Cell 2010; 142: 531-543.

16. Zimmers TA, Davies MV, Koniaris LG, Haynes P, Esquela AF, Tomkinson KN et al. Induction of cachexia in mice by systemically administered myostatin. Science 2002; 296: $1486-1488$. 
17. Massague J. TGFbeta in Cancer. Cell 2008; 134: 215-230.

18. Warburg O. On the origin of cancer cells. Science 1956; 123: 309-314.

19. Vander Heiden MG, Cantley LC, Thompson CB. Understanding the Warburg effect: the metabolic requirements of cell proliferation. Science 2009; 324: 1029-1033.

20. Liu X, Hajnoczky G. Altered fusion dynamics underlie unique morphological changes in mitochondria during hypoxia-reoxygenation stress. Cell Death Differ 2011: 18: 1561-1572.

21. Shimizu S, Narita M, Tsujimoto Y. Bcl-2 family proteins regulate the release of apoptogenic cytochrome $\mathrm{c}$ by the mitochondrial channel VDAC. Nature 1999; 399: 483-487.

22. Abu-Hamad S, Sivan S, Shoshan-Barmatz V. The expression level of the voltagedependent anion channel controls life and death of the cell. Proc Natl Acad Sci USA 2006; 103: $5787-5792$.

23. Geula S, Ben-Hail D, Shoshan-Barmatz V. Structure-based analysis of VDAC1: N-terminus location, translocation, channel gating and association with anti-apoptotic proteins. Biochem J 2012; 444: 475-485.

24. Geula S, Naveed H, Liang J, Shoshan-Barmatz V. Structure-based analysis of VDAC1 protein: defining oligomer contact sites. J Biol Chem 2012; 287: 2179-2190.

25. Pastorino JG, Shulga N, Hoek JB. Mitochondrial binding of hexokinase II inhibits Bax-induced cytochrome $c$ release and apoptosis. J Biol Chem 2002; 277: 7610-7618.

26. Azoulay-Zohar H, Israelson A, Abu-Hamad S, Shoshan-Barmatz V. In self-defence: hexokinase promotes voltage-dependent anion channel closure and prevents mitochondria-mediated apoptotic cell death. Biochem J 2004; 377(Pt 2): 347-355.

27. Hresko RC, Heimberg H, Chi MM, Mueckler M. Glucosamine-induced insulin resistance in 3T3-L1 adipocytes is caused by depletion of intracellular ATP. J Biol Chem 1998; 273 20658-20668

28. Binyamin L, Assaraf YG, Reiter Y. Probing ATP-dependent conformational changes in the multidrug resistance protein 1 (MRP1/ABCC1) in live tumor cells with a novel recombinant single-chain Fv antibody targeted to the extracellular N-terminus. Int J Cancer 2005; 116 703-709.

29. Rathmell JC, Fox CJ, Plas DR, Hammerman PS, Cinalli RM, Thompson CB. Akt-directed glucose metabolism can prevent Bax conformation change and promote growth factorindependent survival. Mol Cell Biol 2003; 23: 7315-7328.

30. Majewski N, Nogueira V, Bhaskar P, Coy PE, Skeen JE, Gottlob K et al. Hexokinasemitochondria interaction mediated by Akt is required to inhibit apoptosis in the presence or absence of Bax and Bak. Mol Cell 2004; 16: 819-830.

31. Kim BC, Mamura M, Choi KS, Calabretta B, Kim SJ. Transforming growth factor beta 1 induces apoptosis through cleavage of $\mathrm{BAD}$ in a Smad3-dependent mechanism in $\mathrm{FaO}$ hepatoma cells. Mol Cell Biol 2002; 22: 1369-1378.

32. Sanchez-Capelo A. Dual role for TGF-beta1 in apoptosis. Cytokine Growth Factor Rev 2005; 16: 15-34.

33. Shoshan-Barmatz V, Golan M. Mitochondrial VDAC1: function in cell life and death and target for cancer therapy. Curr Med Chem 2012; 19: 714-735.

34. Baines CP, Kaiser RA, Sheiko T, Craigen WJ, Molkentin JD. Voltage-dependent anion channels are dispensable for mitochondrial-dependent cell death. Nat Cell Biol 2007; 9: 550-555.

35. Green DR, Kroemer G. The pathophysiology of mitochondrial cell death. Science 2004; 305: 626-629.

36. Zheng $\mathrm{Y}$, Shi $\mathrm{Y}$, Tian $\mathrm{C}$, Jiang $\mathrm{C}$, Jin $\mathrm{H}$, Chen J et al. Essential role of the voltagedependent anion channel (VDAC) in mitochondrial permeability transition pore opening and cytochrome c release induced by arsenic trioxide. Oncogene 2004; 23: 1239-1247.

37. Tsujimoto Y, Shimizu S. VDAC regulation by the Bcl-2 family of proteins. Cell Death Diffe 2000; 7: 1174-1181.

38. Pastorino JG, Hoek JB. Regulation of hexokinase binding to VDAC. J Bioenerg Biomembr 2008; 40: 171-182.
39. Pastorino JG, Hoek JB, Shulga N. Activation of glycogen synthase kinase 3beta disrupts the binding of hexokinase II to mitochondria by phosphorylating voltage-dependent anion channel and potentiates chemotherapy-induced cytotoxicity. Cancer Res 2005; 65: 10545-10554.

40. Mergenthaler P, Kahl A, Kamitz A, van Laak V, Stohlmann K, Thomsen S et al. Mitochondrial hexokinase II (HKII) and phosphoprotein enriched in astrocytes (PEA15) form a molecular switch governing cellular fate depending on the metabolic state. Proc Natl Acad Sci USA 2012; 109: 1518-1523.

41. Banerjee J, Ghosh S. Phosphorylation of rat brain mitochondrial voltage-dependent anion as a potential tool to control leakage of cytochrome c. J Neurochem 2006; 98: 670-676.

42. Yuan S, Fu Y, Wang X, Shi H, Huang Y, Song X et al. Voltage-dependent anion channel 1 is involved in endostatin-induced endothelial cell apoptosis. FASEB J 2008; 22 2809-2820.

43. Murphy KT, Chee A, Gleeson BG, Naim T, Swiderski K, Koopman R et al. Antibodydirected myostatin inhibition enhances muscle mass and function in tumor-bearing mice. Am J Physiol Regul Integr Comp Physiol 2011; 301: R716-R726.

44. Tsuchida K. Activins, myostatin and related TGF-beta family members as novel therapeutic targets for endocrine, metabolic and immune disorders. Curr Drug Targets Immune Endocr Metabol Disord 2004; 4: 157-166.

45. Rosano C. Molecular model of hexokinase binding to the outer mitochondrial membrane porin (VDAC1): Implication for the design of new cancer therapies. Mitochondrion May 2011; 11: 513-519.

46. Galluzzi L, Kepp O, Tajeddine N, Kroemer G. Disruption of the hexokinase-VDAC complex for tumor therapy. Oncogene 2008; 27: 4633-4635

47. Shoshan-Barmatz V, Golan M. Mitochondrial VDAC1: function in cell life and death and a target for cancer therapy. Curr Med Chem 2012; 19: 714-735.

48. Cannata DJ, Ireland Z, Dickinson H, Snow RJ, Russell AP, West JM et al. Maternal creatine supplementation from mid-pregnancy protects the newborn spiny mouse diaphragm from intrapartum hypoxia-induced damage. Pediatr Res 2010; 68: 393-398.

49. Yang W, Zhang Y, Ma G, Zhao X, Chen Y, Zhu D. Identification of gene expression modifications in myostatin-stimulated myoblasts. Biochem Biophys Res Commun 2005; 326: 660-666.

50. Zheng $\mathrm{Y}$, Yamaguchi $\mathrm{H}$, Tian $\mathrm{C}$, Lee MW, Tang $\mathrm{H}$, Wang $\mathrm{HG}$ et al. Arsenic trioxide (As(2) $\mathrm{O}(3)$ ) induces apoptosis through activation of Bax in hematopoietic cells. Oncogene 2005; 24: 3339-3347.

51. Zhu Y, Zhao L, Liu L, Gao P, Tian W, Wang X et al. Beclin 1 cleavage by caspase-3 inactivates autophagy and promotes apoptosis. Protein Cell 2010; 1: 468-477.

52. Ni B, Ma Q, Li B, Zhao L, Liu Y, Zhu Y et al. Phenylarsine oxide induces apoptosis in Baxand Bak-deficient cells through upregulation of Bim. Clin Cancer Res 2012; 18: 140-151.

53. Pon LA, Schon EA. Mitochondria. 2nd edn. Academic Press: Amsterdam; Boston, 2007. xxiv, 920 p.pp.

54. Wu M, Neilson A, Swift AL, Moran R, Tamagnine J, Parslow D et al. Multiparameter metabolic analysis reveals a close link between attenuated mitochondrial bioenergetic function and enhanced glycolysis dependency in human tumor cells. Am J Physiol Cell Physiol 2007; 292: C125-C136.

Cell Death and Disease is an open-access journal published by Nature Publishing Group. This work is licensed under the Creative Commons Attribution-NonCommercialShare Alike 3.0 Unported License. To view a copy of this license, visit http://creativecommons.org/licenses/by-nc-sa/3.0/ 